\title{
Rapamycin-induced autophagy activity promotes bone fracture healing in rats
}

\author{
GE YANG, XUNHONG DUAN, DASHENG LIN, TEN LI, DEQING LUO, LEI WANG and KEJIAN LIAN \\ Department of Orthopedic Surgery, The Affiliated Southeast Hospital of Xiamen University, \\ Orthopaedic Center of People's Liberation Army, Zhangzhou, Fujian 363000, P.R. China
}

Received August 23, 2014; Accepted June 1, 2015

DOI: $10.3892 /$ etm.2015.2660

\begin{abstract}
Autophagy is a crucial mediating process for normal bone cell function and metabolism in physiology or pathology. Rapamycin has been demonstrated to induce the autophagy pathway by inhibiting the mammalian target of rapamycin (mTOR) pathway. However, the contribution of autophagy in orthopedic diseases is rarely reported. The aim of the present study was to evaluate the capacity of pharmacologically induced autophagy to modify disease function in a rat model of bone fracture. A femur fracture model was established via surgery in adult male Sprague-Dawley rats. Rapamycin ( $n=63$ rats) or dimethyl sulfoxide (DMSO) vehicle control ( $n=63$ rats) was administered intraperitoneally for 2, 4 and 6 weeks, and 21 randomly selected rats were sacrificed in each group at each time point. X-ray micro-computed tomography and hematoxylin and eosin staining were used to evaluate the extent of fracture healing in each group. The effects of rapamycin on autophagy, mTOR signaling and the expression levels of vascular endothelial growth factor (VEGF) and proliferating cell nuclear antigen (PCNA) were analyzed using immunohistochemistry, immunofluorescence staining and western blot analysis. Rapamycin affected the mTOR signaling pathway in rats following fracture, as indicated by the inhibition of the phosphorylation of ribosomal protein S6, a target of mTOR, and activation of microtubule-associated protein 2 light chain 3, a key marker of autophagy. Histomorphometry and image examination indicated that the number of osteoblasts in each section was significantly $(\mathrm{P}<0.01)$ increased in the rapamycin group compared with the control group, and this was associated with a significant $(\mathrm{P}<0.05)$ increase in mineralized callus fraction. Furthermore, rapamycin treatment increased the expression levels of VEGF and PCNA in the rat callus tissue. These results suggest that rapamycin may serve a
\end{abstract}

Correspondence to: Dr Kejian Lian, Department of Orthopedic Surgery, The Affiliated Southeast Hospital of Xiamen University, Orthopaedic Center of People's Liberation Army, 269 Zhanghuazhong Road, Zhangzhou, Fujian 363000, P.R. China

E-mail: jiamen88@163.com

Key words: autophagy, rapamycin, bone fracture healing beneficial function in fracture healing, and that the underlying mechanism may involve the activation of autophagy.

\section{Introduction}

Bone fracture is a common injury, which may initiate a series of biophysiological and pathological reactions. These reactions may lead to fracture healing, but may additionally result in tissue damage. A number of promising therapeutic approaches have been developed, such as improvement of internal fixation devices and the application of novel biological materials; however, delayed healing or nonunion may occur in $5-10 \%$ of fractures, adding further to patient morbidity and the expense of treatment (1). The improvement of patient morbidity and reduction of costs is an incentive for the development of novel therapies to enhance fracture healing (2).

Autophagy is the process of cellular 'self-digestion', and serves an essential role in energy and nutrient regulation, in addition to the removal of damaged and dysfunctional macromolecules and organelles $(3,4)$. At the cellular level, failure of autophagy results in the increased expression of abnormal genes, and may lead to cell death (5). Potential consequences of autophagy failure at the tissue and organismal level include neurodegeneration, cardiomyopathies, abnormal skeletal development and premature mortality (6-8). Microtubule-associated protein 2 light chain 3 (LC3-II) is considered to be a primary marker of autophagy (9).

The mammalian target of rapamycin (mTOR) complex is a crucial suppressor of autophagy, functioning upstream of the autophagy-related proteins, and is centrally regulated by multiple upstream signaling pathways involving phosphatidylinositol-3-kinase (PI-3K)/Akt and adenosine monophosphate-activated protein kinase (10). Furthermore, imbalances in the mTOR pathway are involved in cardiac hypertrophy, inflammatory diseases, and diabetes, and pharmacological intervention of mTOR has been proposed as a potential treatment for these conditions (11). Rapamycin is a lipophilic macrolide antibiotic that is used as an immunosuppressive drug in solid organ transplantation, and is able to induce autophagy by inhibiting ribosomal protein S6 (rpS6), a downstream target of mTOR complex 1 (mTORC1) phosphorylation $(12,13)$. In addition, rapamycin treatment has been demonstrated to extend lifespan in mice (14), and protects against aging-associated pathologies of the brain and 
heart (15-18). However, to the best of our knowledge no prior studies have investigated the effects of autophagy on bone fracture healing following an intervention affecting the mTOR pathway.

Bone fractures may impair cellular homeostasis and induce significant stress in bone cells, leading to the activation of the autophagy pathway. Therefore, the aim of the present study was to investigate the effects of the pharmacological enhancement of autophagy on the process of experimental fracture healing in rats.

\section{Materials and methods}

The study was performed in accordance with protocols of the local governmental animal care committee and the Institutional Animal Care and Use Committee at Xiamen University (Zhangzhou, China). Every effort was made to minimize animal suffering and to reduce the number of animals used.

Experimental groups and surgical procedure. A total of 126 adult male Sprague-Dawley rats weighing 250-270 g were obtained from Xiamen University. A total of 63 rats in the rapamycin group received a daily intraperitoneal injection of rapamycin ( $1 \mathrm{mg} / \mathrm{kg}$ body weight/dose) from the day of fracture until they were sacrificed at 2, 4 or 6 weeks after fracture. Subsequently, the degree of fracture healing was analyzed using radiological ( $n=15$ each at 2, 4 and 6 weeks), hematoxylin and eosin (H\&E) staining $(n=12)$, western blot analysis $(n=12)$, immunohistochemistry $(n=12)$ and immunofluorescence $(n=12)$ methods. Rats in the vehicle-treated control group $(n=63)$ received $0.4 \%$ dimethyl sulfoxide (DMSO) in a total injection volume of $0.3 \mathrm{ml}$. Among these animals, fracture healing was analyzed using radiological $(n=15$ each at 2, 4 and 6 weeks), H\&E staining ( $n=12$ each), western blot analysis $(n=12$ each), immunohistochemistry $(n=12$ each) and immunofluorescence $(n=12$ each) methods. For surgery, the rats were anesthetized by intraperitoneal injection of ketamine ( $75 \mathrm{mg} / \mathrm{kg}$ ) and xylazine $(25 \mathrm{mg} / \mathrm{kg})$, which was provided by the Affiliated Southeast Hospital of Xiamen University. The right femur of each animal was exposed and a wire saw was used to make a middle transverse fracture, which was stabilized using a 1.0-mm diameter Kirschner wire as described previously (19). The resulting fracture was of type A3.2, according to the Müller AO classification of fractures (20). X-ray imaging (Multix TOP; Siemens, Forchheim, Germany) was used to document the positions of the implants.

X-ray radiography and micro-computed tomography (microCT) imaging. Fractured limbs were observed at 2, 4 and 6 weeks by performing posteroanterior X-ray radiography to record callus formation. Subsequently, fractured limbs were dissected free of soft tissues, and the intramedullary Kirschner wires were extracted to facilitate scanning using a $\mu$ CT 40 micro-CT device (Scanco Medical AG, Brüttisellen, Switzerland) at 2,800 views, 5 frames per view, $35 \mathrm{kV}$ and $35 \mu \mathrm{A}$. Three-dimensional (3D) images were rendered, and the areas of the transverse section and of void spots in each transverse section image were evaluated using VGStudio MAX software (Dürr, Bietigheim-Bissingen, Germany). The fraction of mineralized callus was quantified by calculating the total void area (which indicates the degree of residual non-mineralized tissue) as a percentage of the total area of the transverse section image.

Histomorphometric analyses. Fractured limbs were harvested and fixed in $4 \%$ formalin for $24 \mathrm{~h}$, decalcified in $10 \%$ ethylenediaminetetraacetic acid solution for 1 month, and embedded in paraffin for histological analysis. Decalcified longitudinal sections were cut to 4-5 $\mu \mathrm{m}$, stained with $\mathrm{H} \& \mathrm{E}$ and the total number of osteoblasts in each callus section was counted.

Immunohistochemistry. Sections from paraffin-embedded samples were deparaffinized using the xylene substitute Pro-Par Clearant (Anatech, Ltd., Battle Creek, MI, USA) and rehydrated in graded ethanol and water. For antigen unmasking, sections in $10 \mathrm{mM}$ sodium citrate buffer ( $\mathrm{pH} \mathrm{6.0)}$ were heated in a microwave oven at $80-85^{\circ} \mathrm{C}$ for $2 \mathrm{~min}$. Slides were then cooled at room temperature for $30 \mathrm{~min}$. After washing with phosphate-buffered saline (PBS), sections were blocked with $5 \%$ serum for $30 \mathrm{~min}$ at room temperature. Polyclonal anti-phospho-rpS6 primary antibody (1:100; Abcam, Cambridge, UK) was applied and the sections were incubated overnight at $4^{\circ} \mathrm{C}$. After washing with PBS, sections were incubated with polyclonal biotinylated goat anti-rabbit secondary antibody (1:100; Abcam) for $1 \mathrm{~h}$ at $37^{\circ} \mathrm{C}$ and incubated with Vectastain ABC-alkaline phosphatase (AP) (Vector Laboratories, Inc., Burlingame, CA, USA) for $30 \mathrm{~min}$. Slides were washed 3 times with PBS, and sections were incubated with AP substrate for $30 \mathrm{~min}$.

Immunofluorescence. Paraffin-embedded samples were deparaffinized using Pro-Par Clearant and rehydrated in graded ethanol and water. After washing with PBS, sections were blocked with $5 \%$ serum for $30 \mathrm{~min}$ at room temperature, then incubated with rabbit anti-LC3-II polyclonal antibody (1:150; Abcam) overnight at $4^{\circ} \mathrm{C}$. After washing with PBS, sections were incubated with polyclonal Alexa Fluor 488 anti-rabbit IgG secondary antibody (1:200; Abcam) for $1 \mathrm{~h}$. Finally, slides were washed and mounted with ProLong Gold Antifade Reagent (Invitrogen Life Technologies, Carlsbad, CA, USA).

Western blot analysis. Rats were anesthetized and the soft tissue covering the diaphyseal part of the femora was removed prior to sacrifice. The visible callus was resected and frozen in nitrogen. For extraction of the whole-protein fraction, tissue samples were homogenized in $250 \mu 1$ radioimmunoprecipitation assay buffer, containing $150 \mathrm{mM} \mathrm{NaCl}, 1 \% \mathrm{NP}-40,25 \mathrm{mM}$ Tris- $\mathrm{HCl}$ (pH 7.6), $0.1 \%$ sodium dodecyl sulfate polyacrylamide (SDS), $1 \%$ sodium deoxycholate and a protease inhibitor cocktail (Abcam). Next, the samples were incubated for $30 \mathrm{~min}$ on ice and centrifuged for $30 \mathrm{~min}$ at $16,000 \mathrm{x} \mathrm{g}$. The supernatant was saved as a whole-protein fraction, and the protein concentration was by Lowry assay. Subsequently, $60 \mathrm{mg}$ protein per lane was separated on $10 \%$ SDS gel and transferred to a polyvinyldifluoride membrane (Thermo Scientific, Waltham, MA, USA). After blocking with 5\% skimmed milk solution for $1 \mathrm{~h}$, membranes were incubated for $2 \mathrm{~h}$ with $0.5 \mu \mathrm{g} / \mathrm{ml}$ rat monoclonal anti-VEGF (Abcam) or $0.5 \mu \mathrm{g} / \mathrm{ml}$ anti-PCNA (Abcam) antibodies. Antibody-protein complexes were visualized using 

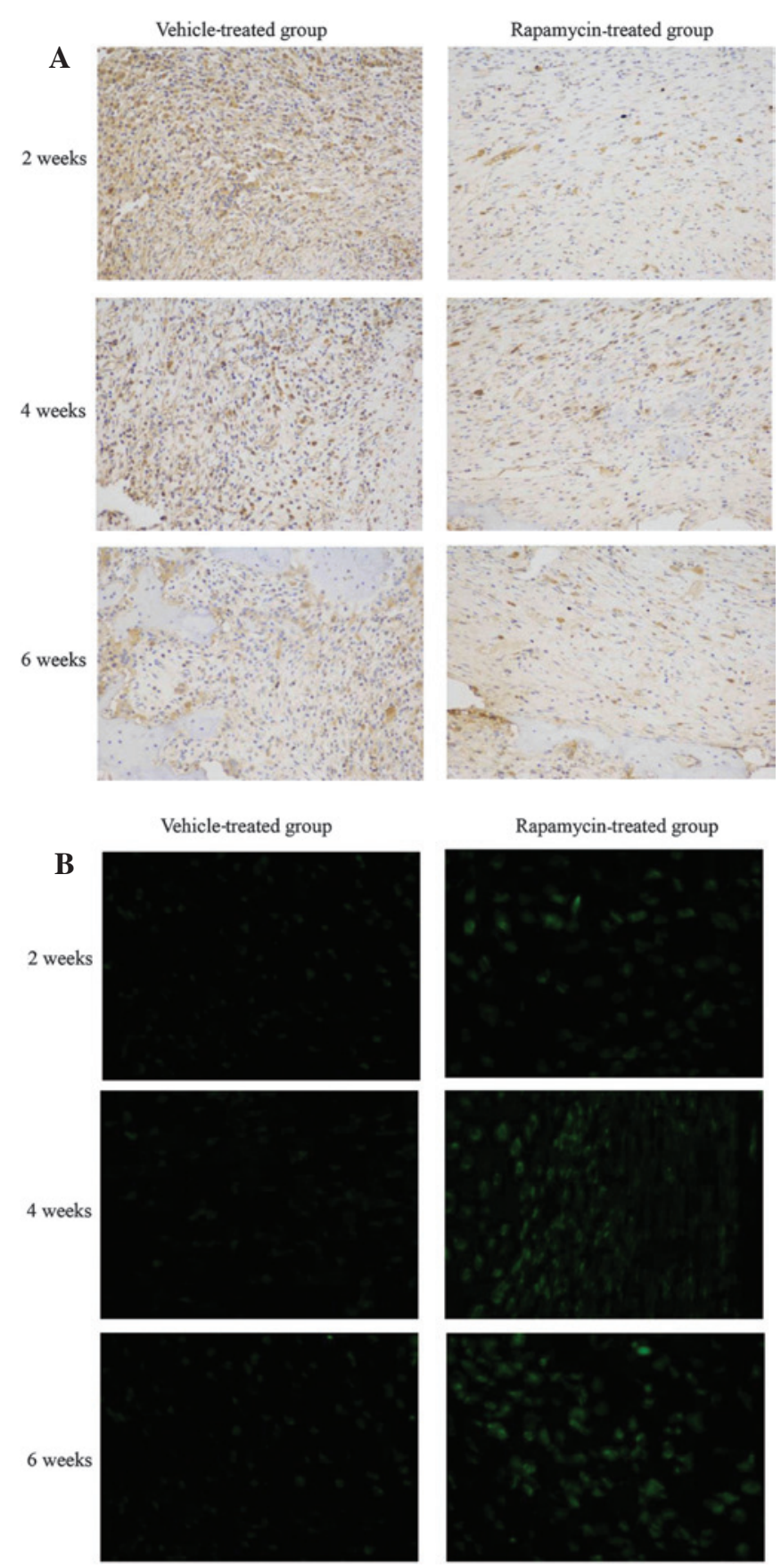

Figure 1. Systemic administration of rapamycin modulates the mammalian target of rapamycin signaling pathway and autophagy in a rat fracture model. Calluses from rats were collected at 2, 4 and 6 weeks post-fracture after treatment with rapamycin or the vehicle ( $n=12$ per group). (A) Sections were analyzed using immunohistochemistry for phosphorylation of ribosoma protein S6. (B) Sections were analyzed by immunofluorescence for light chain 3-II (magnification, x200).

chemiluminescence (ProLong Gold Antifade Reagent) and photographs of the specific protein bands were captured with a Fusion FX7 imaging system (Vilber Lourmat Deutschland GmbH, Eberhardzell, Germany). Quantity One software (Bio-Rad Laboratories GmbH, Munich, Germany) was used for the analysis and quantification of the images. Glyceraldehyde 3-phosphate dehydrogenase was used as an internal control.

Statistical analysis. Results are presented as the mean \pm standard deviation. Comparisons between two groups were conducted

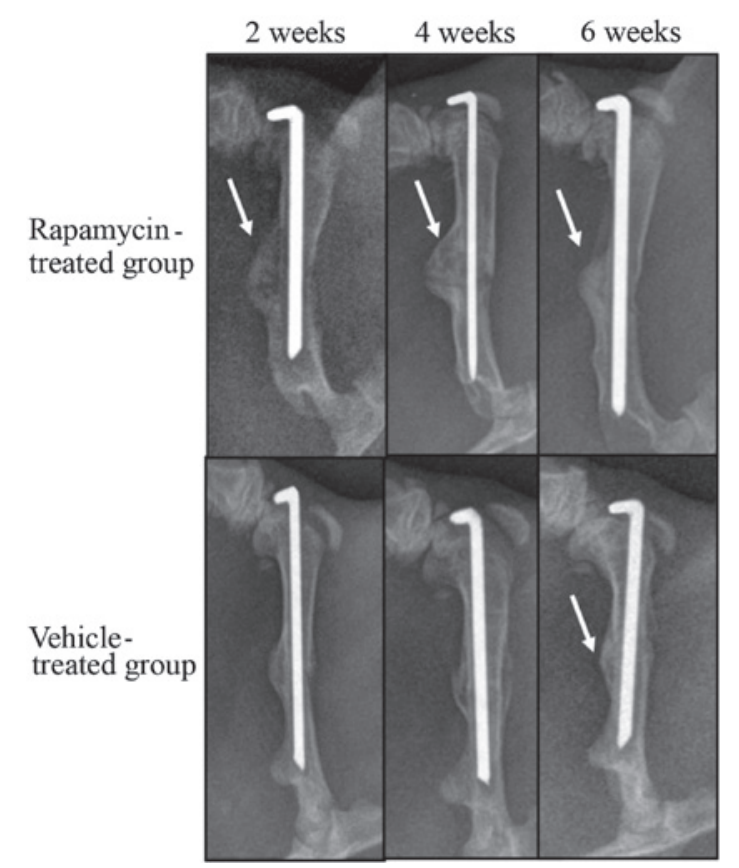

Figure 2. Effect of rapamycin on the size and mineralization of fracture calluses during bone repair. Representative X-ray images of fracture calluses from rapamycin and vehicle-treated rats at 2, 4 and 6 weeks post-fracture. Arrow indicates density of mineralization with fracture calluses.

with t-tests. Statistical analysis was performed using SPSS version 20.0 (IBM, Armonk, NY, USA). P<0.05 was considered to indicate a statistically significant difference.

\section{Results}

Systemic administration of rapamycin modulates mTOR signaling and autophagy in a rat fracture model. The phosphorylation levels of rpS6, a downstream target of mTORC1 (21), were determined to evaluate the effect of rapamycin on the mTOR signaling pathway in a rat femur fracture model. Rapamycin treatment suppressed rpS6 phosphorylation in bone tissue cells in the callus compared with that in vehicle-treated rats (Fig. 1A). In order to determine whether autophagy is promoted as a result of mTOR inhibition by rapamycin, bone tissue sections were stained with LC3-II antibody. An increase in LC3-II expression was detected following rapamycin treatment. This increase correlated with an increase in LC3-II puncta, indicating a marked activation of autophagy in the bone tissue (Fig. 1B). These results indicate that mTOR signaling and autophagy were induced in bone tissue by the intraperitoneal administration of rapamycin.

Rapamycin promotes callus formation and remodeling. To investigate the role of $\mathrm{mTOR}$ in fracture repair, radiographic analyses of the healing bones were conducted at 2, 4 and 6 weeks after fracture (Fig. 2). The calluses from the rats in the vehicle-treated group were not mineralized at 2 weeks after fracture. At 4 weeks, the calluses in this group exhibited a higher mineral density. At 6 weeks post-fracture, calluses from the vehicle-treated group appeared to have been resorbed, as indicated by their reduced size compared with that at week 4 . In contrast with the vehicle-treated group, fracture calluses 
A

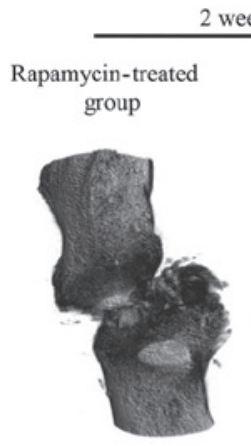

B

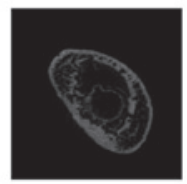

2 weeks

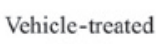

group
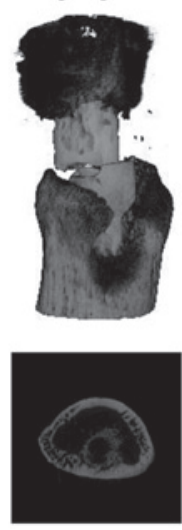

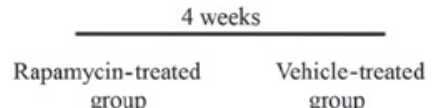

group
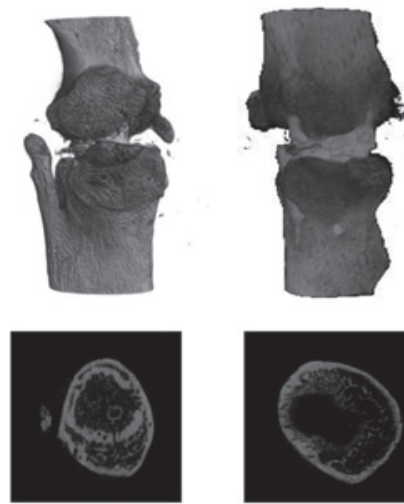
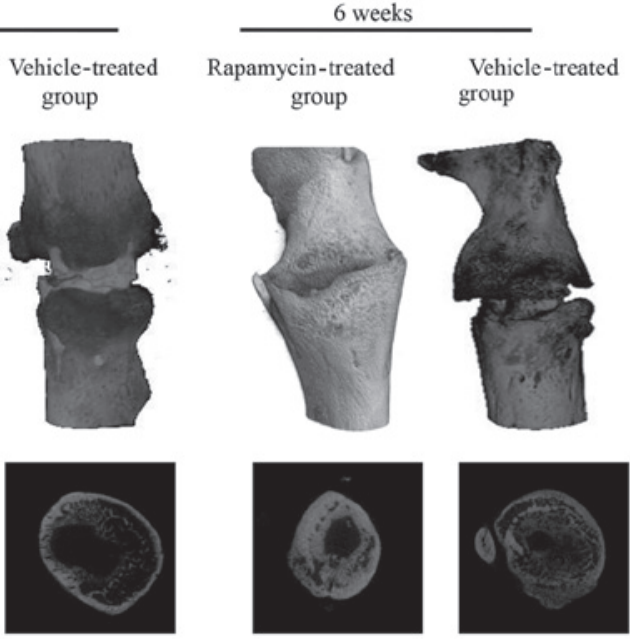

C

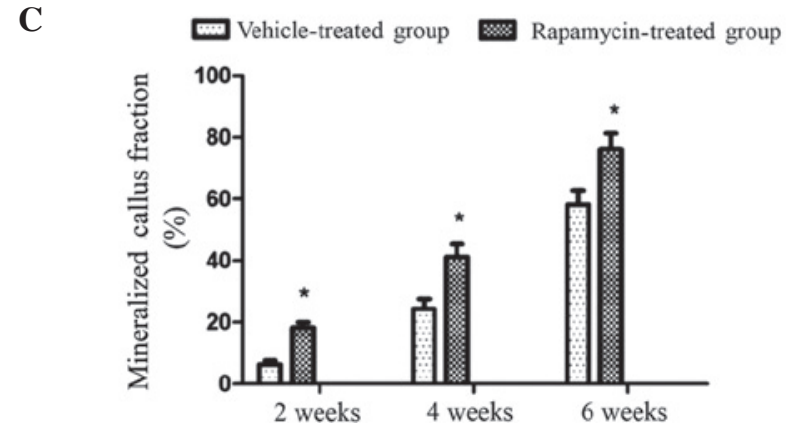

Figure 3. Influence of rapamycin on fracture callus mineralization and remodeling. Bone fracture global imaging was used to rebuild bone structure by micro-computed tomography (micro-CT). All fracture callus sites from rapamycin- and vehicle-treated rats were harvested at 2, 4 and 6 weeks post-fracture ( $n=15$ per group). (A and B) Global image and transverse section of fracture calluses were scanned and graphically re-constructed using micro-CT. (C) Mineralized callus fraction was analyzed using micro-CT. Values are presented as the mean \pm standard deviation. ${ }^{*} \mathrm{P}<0.05$ vs. the vehicle-treated group.

from the rapamycin-treated group were mineralized at 2 weeks post-fracture. By week 4 , the calluses from the rapamycin group were increased in size compared with those from the vehicle-treated group. The calluses from the rapamycin group closely resembled those from the vehicle-treated group at 6 weeks, with resorption of the calluses; however, the mineral density was increased in the rapamycin group compared with that in the vehicle-treated group.

Micro-CT imaging was used to remodel the fracture calluses and to quantify the degree of mineralization at 2,4 and 6 weeks post-fracture (Fig. 3A). 3D representations of the fracture showed that at 2, 4 and 6 weeks post-fracture, the mineralized fracture calluses in the rapamycin group were larger than those in the vehicle-treated group. Transverse sections demonstrated that the rapamycin group exhibited mineralized fracture calluses with a higher degree of mineralization compared with those in the vehicle-treated group at all three time points (Fig. 3B). Quantitative analysis, which involved determining the total percentage area of void regions in the transverse section image indicated that the fraction of mineralized callus in the rapamycin group rats was increased with time by $18.3 \pm 1.6$, $41.2 \pm 4.1$ and $76.2 \pm 5.1 \%$ at 2,4 and 6 weeks post-fracture, respectively. These values are significantly increased compared with those in the vehicle-treated group at each time point (Fig. 3C). These data indicate that treatment with rapamycin is able to enhance callus mineralization and promote callus formation and remodeling in this rat fracture model.
Rapamycin increases the number of osteoblasts in the rat callus, and the expression levels of PCNA and VEGF in cells. To investigate the mechanism of action of rapamycin in the rats, a total of four sections were observed for each group at each time point to analyze the osteoblast cell density in the callus. It was observed that osteoblast formation occurred at a greater extent in the rats of the rapamycin group at the three time points post-fracture (Fig. 4A), and the difference in osteoblast number between the two groups was statistically significant $(\mathrm{P}<0.01$; Fig. 4B).

Western blot analysis was performed to determine the expression levels of PCNA and VEGF. PCNA is a crucial protein for the proliferation of osteoblasts, and VEGF is known to be a crucial molecule in the promotion of angiogenesis during fracture healing. Increased expression levels of PCNA and VEGF were detected in the rapamycin group rats compared with the vehicle-treated rats at each time point. This increase was statistically significant $(\mathrm{P}<0.05$; Fig. 5$)$. This finding indicates that rapamycin contributes to osteoblast proliferation by promoting PCNA and callus blood supply through the promotion of VEGF expression.

\section{Discussion}

There is evidence to suggest that effective autophagy exists within a narrow homeostatic range to regulate protein homeostasis and cell survival (22). To a certain extent, autophagy 


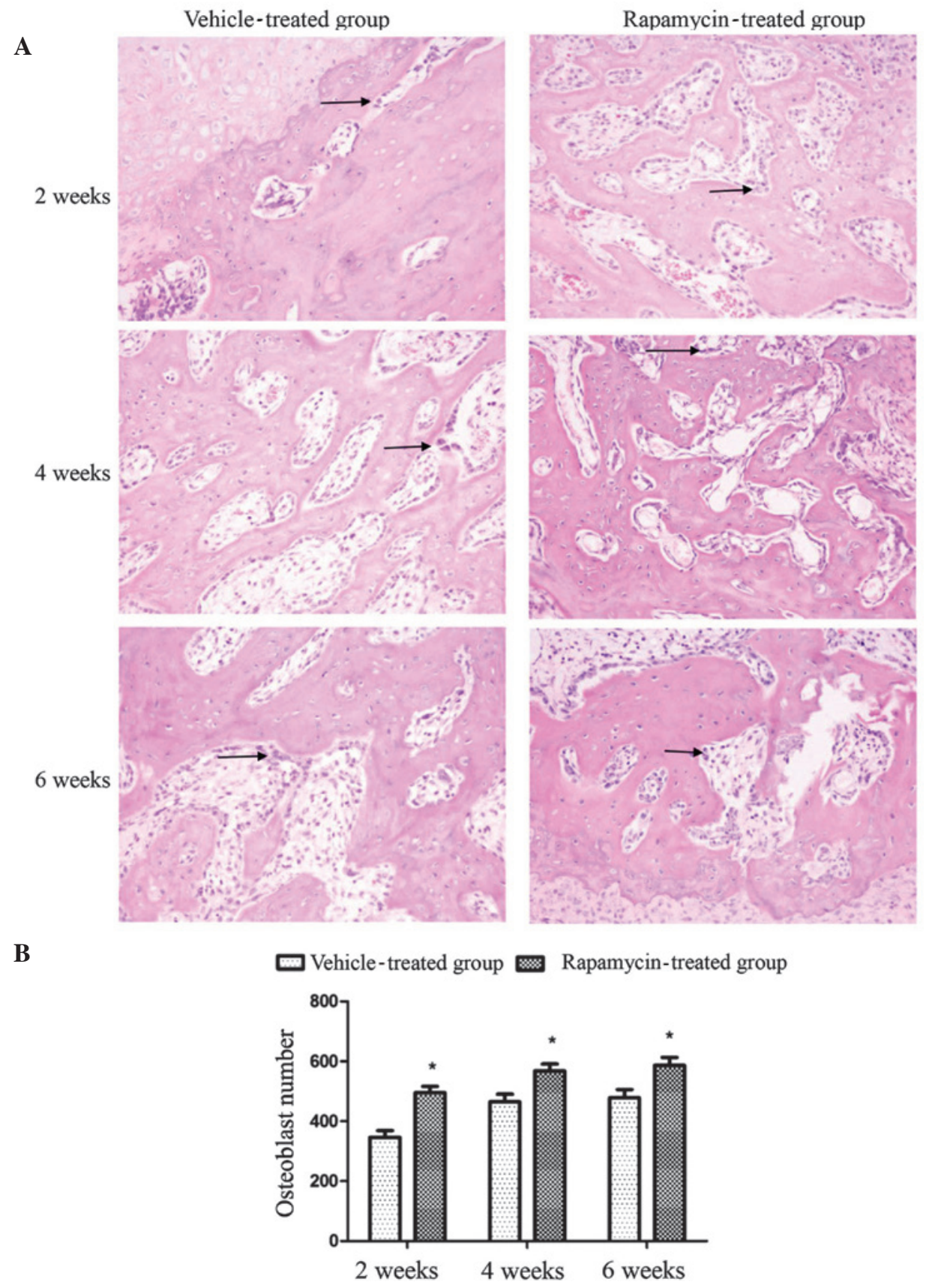

Figure 4. Rapamycin increase the number of osteoblasts in the rat callus. (A) Calluses section from Sprague-Dawley rats at 2, 4 and 6 weeks post-fracture under treatment with rapamycin or vehicle were stained with hematoxylin and eosin ( $\mathrm{n}=12$ per group; magnification, $\mathrm{x} 200)$. Arrow indicates an osteoblast in the callus. (B) Quantitative analysis of osteoblast number indicated a significant increase in cellularity after rapamycin treatment compared with the vehicle treatment. Values are presented as the mean \pm standard deviation. ${ }^{*} \mathrm{P}<0.01$ vs. vehicle-treated group.

affects bone formation or loss $(23,24)$. However, to the best of our knowledge there are no previous studies regarding the effects of autophagy on bone repair. On this basis, the aim of the present study was to investigate whether the activation of autophagy affects early bone fracture healing. Rapamycin was selected as an inhibitor of the mTOR signaling pathway, which regulates the initiation of autophagy. Rapamycin is a lipophilic macrolide antibiotic that is used as an immunosuppressive drug and to induce autophagy in a variety of cell types (12-18).

The results of the present study suggest that the systemic administration of rapamycin inhibits the mTOR signaling pathway in the rat callus, as indicated by the reduced phosphorylation of $\mathrm{rpS6}$, which integrates the processes of protein translation with cell growth and proliferation (25). Treatment of cells with rapamycin blocks S6 kinase 1 (S6K1) phosphorylation, and inhibits the activation of S6K1 (26). Furthermore, rapamycin-activated autophagy was indicated by increased expression levels of LC3-II, the most specific autophagosomal marker in the rat callus. These results confirm that the systemic administration of rapamycin modulates mTOR signaling and autophagy in a rat fracture model.

In the present study, treatment with rapamycin was observed to induce a significant improvement in callus formation and mineralization. In fracture healing, the fraction of mineralized callus is an important parameter, which indicates the presence of newly formed bone and the effect of 
A

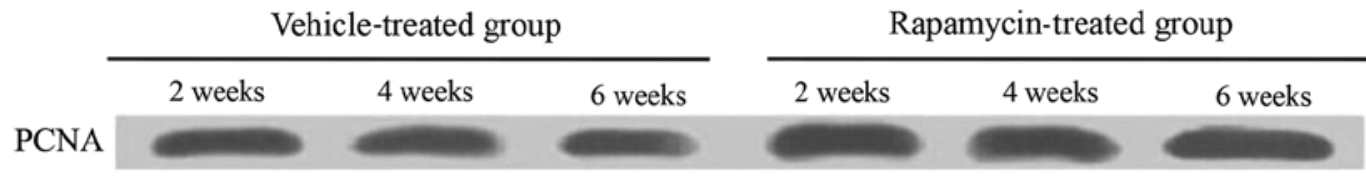
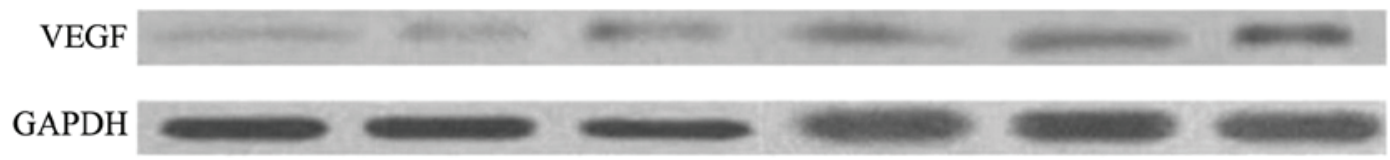

B

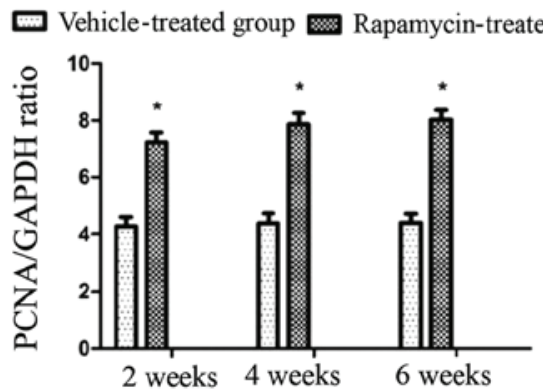

四 Vehicle-treated group $\approx$ Rapamycin-treated group

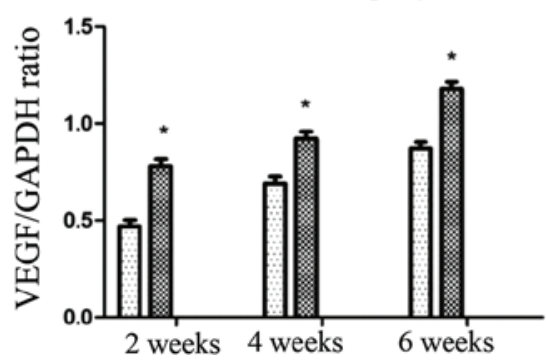

Figure 5. Effects of rapamycin on PCNA and VEGF expression in the callus. (A) Western blot analysis of PCNA and VEGF expression levels in the rat callus at 2, 4 and 6 weeks post-fracture. (B) Quantitative comparison of PCNA and VEGF expression levels between vehicle-treated and rapamycin groups, expressed as a ratio of GAPDH, used as loading control ( $\mathrm{n}=12$ per group). Values are presented as the mean \pm standard deviation. * $\mathrm{P}<0.05$ vs. vehicle-treated group. PCNA, proliferating cell nuclear antigen; VEGF, vascular endothelial growth factor; GAPDH, glyceraldehyde 3-phosphate dehydrogenase.

bone formation or remodeling. Surface calluses formed more rapidly in the rapamycin group than in the vehicle-treated group. Furthermore, mineralization in the fracture calluses of the rapamycin group was significantly increased compared with that in the vehicle-treated group at 2, 4 and 6 weeks post-fracture.

On the basis of the established efficacy of rapamycin in the promotion of callus formation, the expression of VEGF was investigated, as a key growth factor for the promotion of endochondral ossification during secondary fracture healing. This stage of fracture healing comprises: i) Osteocyte survival and cell death; ii) degradation and calcification of the extracellular matrix; and iii) the formation of new blood vessels and new bone tissue (27). VEGF is considered to exert a direct effect on osteoprogenitor cells by promoting osteoblast differentiation and increasing the mineralization of regenerated bone (28). Therefore, the positive effect of rapamycin on fracture repair may be due to the activation of VEGF expression in callus tissue, as suggested by western blot analysis. The increased expression of VEGF may be associated with altered cell proliferation, as indicated by the elevated PCNA levels in the calluses of the rapamycin group. These results are consistent with a previous study in which rapamycin increased the cellular levels of PCNA (29).

Histological analyses showed that the number of osteoblasts in the callus at different time points differed significantly between the rapamycin and vehicle-treated group rats. This difference may be associated with the increased levels of PCNA; thus, it was hypothesized that the observed effects on fracture healing, VEGF and PCNA expression, and osteoblast activity were a result of the inhibition of mTOR by rapamycin. Rapamycin is a specific inhibitor of mTOR, particularly for the mTORC1 protein, and there is no evidence that it exerts off-target effects on enzymes other than the mTOR kinase $(30,31)$. mTOR inhibition is known to induce autophagy; however, other signaling pathways are affected directly or indirectly by mTOR inhibition, such as the PI-3K/Akt signaling pathway (32). With regard to the role of autophagy activation in the effects observed in the present study, it is possible that rapamycin restores the suppressed autophagy, which a previous study had observed in cartilage and osteoarthritis (33), and as result, inhibits cell death. The induction of cell death is a well-known effect of defective autophagy (34). Therefore, it appears that the preservation of osteoblasts in calluses and the promotion of fracture reparation observed in the present study are attributable to the activation of autophagy.

The promotion of VEGF and PCNA expression in the rapamycin-treated rats may be associated with the increased levels of autophagy and mTORC1 inhibition. Previous studies have observed that decreased levels of autophagy and mTORC1 activation are associated with aging-related bone loss and other pathologies $(35,36)$. Conversely, increased levels of autophagy and a reduction in mTORC1 expression may lead to an extension of lifespan $(37,38)$. However, the increased VEGF and PCNA expression levels observed in the rapamycin-treated rats in the present study are inconsistent with those of a previous study by Holstein et al (39). In this study, Holstein et al observed that rapamycin initially delayed fracture healing and reduced VEGF and PCNA expression. It is plausible that this discrepancy is a result of the different doses of rapamycin used in the two studies, or the different animals. Further studies may be required to investigate the effect of various concentrations of rapamycin on bone healing.

There are a number of limitations to the present study. For example, the effect of rapamycin-induced autophagy on the formation of osteoclasts and osteocytes was not investigated, and the results of animal studies do not always correlate with 
results in humans. However, the most notable limitation of the present study is that rapamycin is a therapeutic immunosuppressant drug, which is used widely to treat cancer and reduce organ transplant rejection. The clinical feasibility of the application of mTOR inhibitors to the treatment of fractures may be enhanced by recent advances in the development of novel rapamycin analogs that exhibit improved safety. Rapamycin analogs that are more specifically targeted may activate autophagy with fewer side-effects (40).

The effects of rapamycin on fracture healing have been well documented by Holstein et al (41); however, these authors did not investigate the phosphorylation of rpS6 and the activation of LC3-II. To the best of our knowledge, the present study is the first to investigate the therapeutic benefits of rapamycin-induced autophagy in an animal model of fracture. Adult rats were subjected to surgical femur fracture stabilization, which is a widely used model. For further preclinical development of autophagy activators, future studies may be required to investigate the effects of autophagy on fracture healing in older animals, in order to more realistically model the human condition.

In summary, rapamycin was used to induce the activation of autophagy in rats and the efficacy of this intervention on fracture healing was investigated. These results indicate that the pharmacological inhibition of mTOR and the induction of autophagy by rapamycin may be an effective therapeutic approach for the promotion of fracture healing.

\section{References}

1. Einhorn T: Enhancement of fracture-healing. J Bone Joint Surg Am 77: 940-956, 1995.

2. O'Neill KR, Stutz CM, Mignemi NA, et al: Micro-computed tomography assessment of the progression of fracture healing in mice. Bone 50: 1357-1367, 2012.

3. Mizushima N, Levine B, Cuervo AM and Klionsky DJ: Autophagy fights disease through cellular self-digestion. Nature 451: 1069-1075, 2008.

4. Levine B and Kroemer G: Autophagy in the pathogenesis of disease. Cell 132: 27-42, 2008

5. Mathew R, Karp CM, Beaudoin B, et al: Autophagy suppresses tumorigenesis through elimination of p62. Cell 137: 1062-1075, 2009.

6. Hara T, Nakamura K, Matsui M, et al: Suppression of basal autophagy in neural cells causes neurodegenerative disease in mice. Nature 441: 885-889, 2006.

7. Komatsu M, Waguri S, Ueno T, et al: Impairment of starvation-induced and constitutive autophagy in Atg7-deficient mice. J Cell Biol 169: 425-434, 2005.

8. Shibata M, Lu T, Furuya T, et al: Regulation of intracellular accumulation of mutant Huntingtin by Beclin 1. J Biol Chem 281: 14474-14485, 2006.

9. Su JC, Tseng PH, Hsu CY, et al: RFX1-dependent activation of SHP-1 induces autophagy by a novel obatoclax derivative in hepatocellular carcinoma cells. Oncotarget 5: 4909-4919, 2014

10. Wullschleger S, Loewith $\mathrm{R}$ and Hall MN: TOR signaling in growth and metabolism. Cell 124: 471-484, 2006.

11. Dann SG, Selvaraj A and Thomas G: mTOR Complex1-S6K1 signaling: At the crossroads of obesity, diabetes and cancer. Trends Mol Med 13: 252-259, 2007.

12. Shigemitsu K, Tsujishita Y, Hara K, Nanahoshi M, Avruch J and Yonezawa K: Regulation of translational effectors by amino acid and mammalian target of rapamycin signaling pathways. Possible involvement of autophagy in cultured hepatoma cells. J Biol Chem 274: 1058-1065, 1999.

13. Sabers CJ, Martin MM, Brunn GJ, et al: Isolation of a protein target of the FKBP12-rapamycin complex in mammalian cells. J Biol Chem 270: 815-822, 1995.

14. Sarkar S and Rubinsztein DC: Huntington's disease: Degradation of mutant huntingtin by autophagy. FEBS J 275: 4263-4270, 2008

15. Harrison DE, Strong R, Sharp ZD, et al: Rapamycin fed late in life extends lifespan in genetically heterogeneous mice. Nature 460: 392-395, 2009.
16. Pan T, Rawal P, Wu Y, Xie W, Jankovic J and Le W: Rapamycin protects against rotenone-induced apoptosis through autophagy induction. Neuroscience 164: 541-551, 2009.

17. Spilman P, Podlutskaya N, Hart MJ, et al: Inhibition of mTOR by rapamycin abolishes cognitive deficits and reduces amyloid-beta levels in a mouse model of Alzheimer's disease. PLoS One 5: e9979, 2010.

18. Inuzuka Y, Okuda J, Kawashima T, et al: Suppression of phosphoinositide 3-kinase prevents cardiac aging in mice. Circulation 120: 1695-1703, 2009.

19. Holstein JH, Menger MD, Culemann U, Meier C and Pohlemann T: Development of a locking femur nail for mice. J Biomech 40: 215-219, 2007.

20. Müller ME, Nazarian S, Koch P and Schatzker J (eds): The Comprehensive Classification of Fractures of Long Bones. 1st edition. Springer, New York, NY, 1994.

21. Ekim B, Magnuson B, Acosta-Jaquez HA, Keller JA, Feener EP and Fingar DC: mTOR kinase domain phosphorylation promotes mTORC1 signaling, cell growth and cell cycle progression. Mol Cell Biol 31: 2787-2801, 2011.

22. Moscat J and Diaz-Meco MT: p62 at the crossroads of autophagy, apoptosis and cancer. Cell 137: 1001-1004, 2009.

23. Lin NY, Stefanica A and Distler JH: Autophagy: A key pathway of TNF-induced inflammatory bone loss. Autophagy 9: 1253-1255, 2013.

24. Zhang L, Guo YF, Liu YZ, et al: Pathway-based genome-wide association analysis identified the importance of regulation-of-autophagy pathway for ultradistal radius BMD. J Bone Miner Res 25: 1572-1580, 2010.

25. Zoncu R, Efeyan A and Sabatini DM: mTOR: From growth signal integration to cancer, diabetes and ageing. Nat Rev Mol Cell Biol 12: 21-35, 2011.

26. Guertin DA and Sabatini DM: The pharmacology of mTOR inhibition. Sci Signal 2: pe24, 2009.

27. Yu X, Guo Y, Kang Q and Luo C: Effects and mechanisms of mechanical stress on secondary fracture healing. Front Biosci (Landmark Ed) 18: 1344-1348, 2013.

28. Keramaris NC, Calori GM, Nikolaou VS, Schemitsch EH and Giannoudis PV: Fracture vascularity and bone healing: A systematic review of the role of VEGF. Injury 39 (Suppl 2): S45-S57, 2008

29. Morrow PW, Tung HY and Hemmings HC Jr: Rapamycin causes activation of protein phosphatase-2A1 and nuclear translocation of PCNA in CD4 ${ }^{+} \mathrm{T}$ cells. Biochem Biophys Res Commun 323: 645-651, 2004.

30. Kobayashi S, Kishimoto T, Kamata S, Otsuka M, Miyazaki M and Ishikura $\mathrm{H}$ : Rapamycin, a specific inhibitor of the mammalian target of rapamycin, suppresses lymphangiogenesis and lymphatic metastasis. Cancer Sci 98: 726-733, 2007.

31. Kawahara T, Asthana S and Kneteman NM: m-TOR inhibitors: What role in liver transplantation? J Hepatol 55: 1441-1451, 2011.

32. Cirstea D, Hideshima T, Rodig S, et al: Dual inhibition of Akt/mammalian target of rapamycin pathway by nanoparticle albumin-bound-rapamycin and perifosine induces antitumor activity in multiple myeloma. Mol Cancer Ther 9: 963-975, 2010.

33. Caramés B, Taniguchi N, Otsuki S, Blanco FJ and Lotz M: Autophagy is a protective mechanism in normal cartilage and its aging-related loss is linked with cell death and osteoarthritis. Arthritis Rheum 62: 791-801, 2010.

34. Wong E and Cuervo AM: Autophagy gone awry in neurodegenerative diseases. Nat Neurosci 13: 805-811, 2010.

35. Chen K, Yang YH, Jiang SD and Jiang LS: Decreased activity of osteocyte autophagy with aging may contribute to the bone loss in senile population. Histochem Cell Biol 142: 285-295, 2014.

36. Stanfel MN, Shamieh LS, Kaeberlein M and Kennedy BK: The TOR pathway comes of age. Biochim Biophys Acta 1790: 1067-1074, 2009

37. Sciarretta S, Volpe M and Sadoshima J: Mammalian target of rapamycin signaling in cardiac physiology and disease. Circ Res 114: 549-564, 2014.

38. Mirzaei H and Longo VD: Acetyl-CoA synthetase is a conserved regulator of autophagy and life span. Cell Metab 19: 555-557, 2014.

39. Holstein JH, Klein M, Garcia P, et al: Rapamycin affects early fracture healing in mice. Br J Pharmacol 154: 1055-1062, 2008.

40. Wagner R, Mollison KW, Liu L, et al: Rapamycin analogs with reduced systemic exposure. Bioorg Med Chem Lett 15: 5340-5343, 2005

41. Holstein JH, Klein M, Garcia P, Histing T, Culemann U, Pizanis A, Laschke MW, Scheuer C, Meier C, Schorr H, et al: Rapamycin affects early fracture healing in mice. Br J Pharmacol 154: $1055-1062,2008$ 